\title{
Packaging and Storage of Hops (Humulus lupulus) ${ }^{1}$
}

\author{
Sean Campbell and Brian Pearson ${ }^{2}$
}

\section{Introduction}

Hops (Humulus lupulus), a perennial herbaceous plant grown primarily in the United States and Europe for its mature cones, are an essential ingredient to the production of beer. According to the Brewers Association, there were 5,562 breweries within the United States in June 2017, 906 more breweries than recorded in June 2016 (Brewers Association 2017). The craft brewing industry continues to expand rapidly, with an additional 2,739 breweries being planned. Increased craft beer production and demand for craft beer products has resulted in an increased demand for high-quality, locally sourced ingredients. Increased demand for hops has resulted in record-setting production within the United States, and the expansion of hop cultivation in states where hop cultivation had not occurred previously (USDA 2017).

Once harvested, hop cones should be dried as quickly as possible to prevent degradation of organic compounds responsible for hop flavors and aromas. Timely drying ensures hop cones retain high quality and freshness through a reduction of moisture present at harvest (Pearson 2016). Once dried, hop cones may be processed into pellets. Pelletization is required for use with some brewing equipment, but may not be necessary or preferred by all brewers. Regardless, both non-pelletized and pelletized hops require careful packaging and storage to preserve and maximize hop quality.
Considering the influx of hop production in the state, information about packaging and storing hops is needed. This EDIS publication is aimed to help Florida hop growers and hobby brewers understand how to efficiently package and store hops after harvesting.

\section{Packaging}

Hops, like other processed agricultural crops such as coffee or dried fruit, are highly sensitive to some environmental factors, such as moisture, oxygen, and temperature, that may affect their storability. Improper packaging and storage conditions may negatively influence hop quality or their ability to be used in the production of beer. Hops should be packaged and stored to minimize exposure to moisture, light, heat, and oxygen. High moisture encourages the growth of mold and can leave hops unusable. Exposure to light, heat, and oxygen results in the formation of unwanted compounds that produce unpleasant and "cheesy" aromas, a condition referred to "light struck" or "skunked."

To preserve hop quality, hops should be packaged shortly after post-harvest drying to minimize exposure to atmospheric moisture and oxygen. Hop cones or pellets should be placed in tightly sealed bags, with as much air removed as possible. Removal of excess air can be accomplished through a manual pressing technique, vacuum-assisted removal, or through purging with nitrogen gas followed by vacuum-assisted removal. While nitrogen purging is the most effective method, the equipment required is specialized and may be outside the need of small-scale hop

1. This document is ENH1297, one of a series of the Environmental Horticulture Department, UF/IFAS Extension. Original publication date June 2018. Visit the EDIS website at http://edis.ifas.ufl.edu.

2. Sean Campbell, doctoral research assistant; and Brian Pearson, assistant professor; UF/IFAS Extension Mid-Florida Research and Education Center, Apopka, FL 32703.

The Institute of Food and Agricultural Sciences (IFAS) is an Equal Opportunity Institution authorized to provide research, educational information and other services

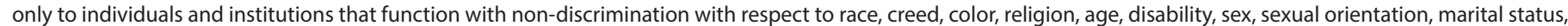

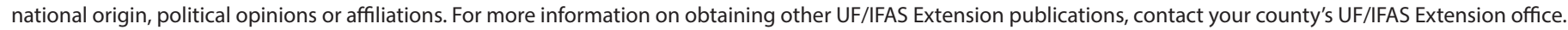
U.S. Department of Agriculture, UF/IFAS Extension Service, University of Florida, IFAS, Florida A \& M University Cooperative Extension Program, and Boards of County Commissioners Cooperating. Nick T. Place, dean for UF/IFAS Extension. 
producers. For those preserving small to medium volumes of hops, a household, food-grade vacuum sealing machine is recommended to properly preserve hops prior to storage.

Although many storage materials are available, the two most popular options recommended for storage of hops are multi-layer plastic and mylar vacuum seal bags. Description of both materials are presented below.

\section{Multi-Layer Plastic Vacuum Seal}

Multi-layer plastic vacuum seal bags work well to block exposure to both moisture and oxygen, and allow users to cut bags to the desired size, thus allowing users to customize package size and reduce material waste. Multi-layer plastic vacuum seal bags are not completely impermeable to oxygen and moisture and, thus, allow a small volume of both to influence the quality of hops over extended storage periods. Rolls of multi-layer plastic vacuum seal bags interface well with household and food-grade vacuum sealing machines. Although these bags allow penetration of light and a very small volume of oxygen and moisture, they are low-cost and widely available. Multi-layer plastic bags are great for packaging low to medium volumes of hops and are recommended as a minimal hop storage packaging material.

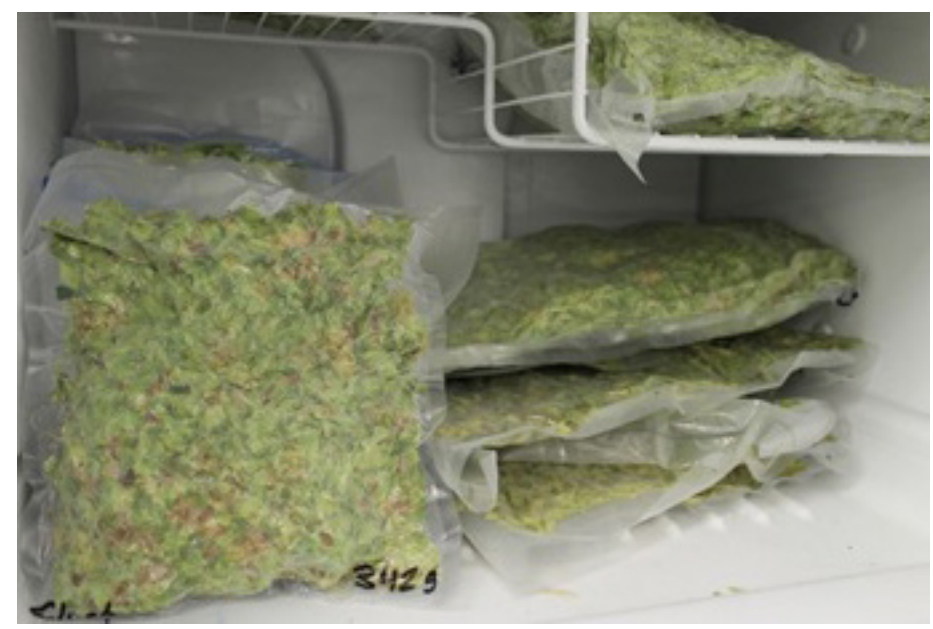

Figure 1. Dried hop cones packaged in multi-layer plastic bags stored in a small-volume, conventional freezer.

Credits: Sean M. Campbell, UF/IFAS

\section{Mylar Foil Vacuum Seal}

Made from reflective foil, mylar vacuum seal bags are superior to multi-layer plastic bags at blocking oxygen and moisture during long-term storage. Given its light-blocking properties, mylar is also much more effective at protecting hops from the negative effects of light, thus allowing hops to be kept in lit storage environments. Mylar, however, does become brittle at low temperatures and may tear easily when stored for extended periods of time in freezers. Mylar foil bags are more expensive than multi-layer plastic and do not allow users the flexibility of cutting bags to size. Mylar bags are most often used with specialized equipment that utilize nitrogen purging for superior long-term hop storage (Homestead Dreamer 2015).

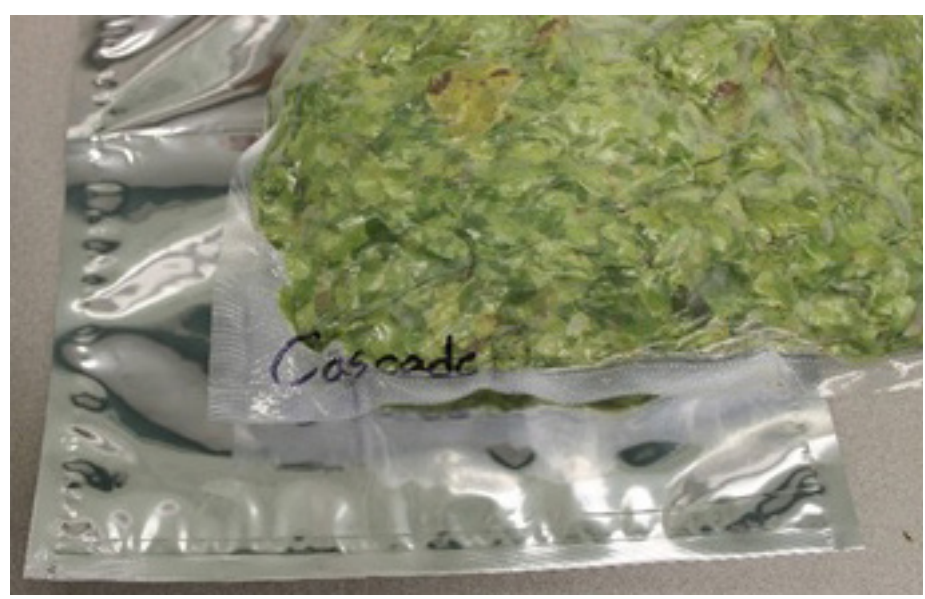

Figure 2. Mylar foil vacuum seal (bottom left), empty; multi-layer plastic vacuum seal (top right), filled with processed whole Cascade hop cones.

Credits: Sean M. Campbell, UF/IFAS

\section{Storage Conditions}

Once packaged, hops should be stored in a cool, dry, dark environment. Storage within a freezer is ideal, as it will slow chemical reactions that degrade hop quality. Light should be eliminated from reaching packaged hops, as it will degrade hop quality with extended periods of exposure. This is important when using multi-layer plastic bags given that such material does not block light from reaching stored hops.

\section{Shelf-Life}

Most producers advocate using hops within a year of harvest; however, when properly packaged and stored within sub-freezing temperatures, hops are considered viable for commercial use for up to five years after harvest. While harvest date can be a good indicator of expected hop quality, stored hops should always be inspected to verify their quality. Properly dried and stored hops should possess a strong aroma and solid green appearance. Once opened, hops should be used promptly to minimize degradation from exposure to environmental conditions.

\section{References}

Sutton, Matt. 2017. "Brewers Association Mid-Year Results Show Stable Growth for Craft Segment." www.brewersassociation.org/press-releases/brewers-association-mid-yearresults-show-stable-growth-craft-segment/ 
Homestead Dreamer. 2015. "Fan Question: Vacuum Sealing vs Mylar." Last modified April 28. http://www.homesteaddreamer.com/2015/04/28/vacuum-sealing-vs-mylar/

Pearson, B. J. 2016. Florida edible garden plants: Hops (Humulus lupulus). ENH1227. Gainesville: University of Florida Institute of Food and Agricultural Sciences. http:// edis.ifas.ufl.edu/ep488

USDA. 2017. "National Hop Report.” https://www.usahops. org/img/blog_pdf/102.pdf 